\title{
A Strategic Behavioral-based Intelligent Transport System with Artificial Immune System *
}

\author{
Henry Y. K. Lau \\ Department of Industrial and Manufacturing \\ Systems Engineering \\ The University of Hong Kong \\ Pokfulam Road Hong Kong \\ hyklau@hkucc.hku.hk
}

\author{
Vicky W.K. Wong \\ Department of Industrial and Manufacturing \\ Systems Engineering \\ The University of Hong Kong \\ Pokfulam Road Hong Kong \\ vickywong@hkusua.hku.hk
}

\begin{abstract}
This paper presents a distributed control framework based on human immunity to manuge, coordinate and schedule a fleet of agents employed ir an automated transport system. Through the study of immunology, each agent is abstracted as an independent agent operating in a multi-agent system that carries local information, searches for solution space and exhibits robust behavior to accomplish tasks. The internal behaviors of the AIS agents, which are decided by their perception of the environment, are studied to describe their strategies in performing various operations. Simulations are presented to examine the significance of each behavioral state in every necessary action or step and the impacts of these states on the overall performance of the transport system. The results of the simulations illustrate the importance of each behavioral state and their inter-relationship in establishing a truly decentralized and non-deterministic transport system.
\end{abstract}

Keywords: Artificial Immune System, automated transport system, behavioral state, agent, cooperation.

\section{Introduction}

The human immune system protects the human boidy from attack by foreign antigens such as viruses and bacteria. It uses specialized organs designed to respond to antigens entering the body's tissues and the mobile force of immune cells in the bloodstream to respond rapidly to attack [1]. The immune system is therefore a highly distributed multi-agent system having complex and sophisticated mechanisms for its regulation. The properties of self-regulation and decentralization impart a high degree of robustness that has created great interest in implementing engineering systems [2-5]. This biological metaphor is known as Artificial Immune System (AIS).

AIS adapts and implements the properties and mechanisms of the human immune system, such as specificity, diversity, memory and discrimination, to perform complicated tasks. These properties are extremely beneficial in developing various engineering systems with the characteristics of self-organization, adaptive control, fault tolerance, memory management and abnormality detection, etc. The theoretical framework of AIS has been widely studied in the field of Artificial Intelligence (AI). Some typical studies include distributed intrusion detection systems [2], autonomous agent controls [3], mine detection systems [4] and fault tolerance systems [5].

Inspired from these appealing properties of AIS, an immunity-based control framework is proposed for multiagent transport systems commonly used for automated material handling. Our control framework is designed for organizing and controlling the autonomous agents that operated as transportation vehicles such as autonomous guided vehicles in a material handling system. These agents are deployed for automated and flexible delivery of cargoes and materials, for example, in container terminals, airport cargo terminals, automated warehouses, hospital complexes and distribution centers. Despite the ultimate usefulness of these agents in fulfilling the task of automated material handling, a generic control for such a multi-agent transport system will have lasting benefits to accommodate and adapt to various operations in the dynamic real-world environment.

This paper presents a distributed control framework based on the biological theory of immunology to manage, coordinate and schedule a fleet of agents employed in an automated transport system. Compared to most existing multi-agent transport systems that utilize a centralized control paradigm, the proposed control framework is fully decentralized. Each agent has its own behaviors and they cooperate via information communication and sharing in order to achieve common goals. Through the study of the human immune system characteristics, each agent is abstracted as an independent agent operating in a multiagent system that carries local information, searches for solution space and exhibits robust behavior to accomplish tasks.

\footnotetext{
"0-7803-8566-7/04/\$20.00 두 2004 IEEE.
} 


\section{Human immune system}

The immunity system protects a human body against foreign antigens by recognizing, eliminating and remembering foreign macromolecules and cell through self and non-self recognition [6]. Immunity is classified into innate immunity and acquired immunity [7]. Innate immunity is inborn and unchanging whereas acquired immunity is developed during the lifetime of a person. Innate immunity provides resistance to a variety of antigens during their first exposure to a human body. This general defense mechanism is known as primary immune response which is slower and less protective. At the same time antigen-specific acquired immune system is activated to eliminate the antigen with a stronger response. The immune cells have special binding areas, known as receptors that can structurally determine and react with specific foreign antigens. When an antigen binds to the immune cells, this interaction sensitizes the proliferation and differentiation of the population of immune cells specific to a particular kind of antigen. Some immune cells will become memory cells for tackling the reoccurrence of the same antigen in the future after the elimination. Hence, a more rapid and stronger response will result in the subsequent exposure to the same antigen. This is called the secondary response.

\section{The AIS-based control framework}

The AIS-based control as depicted by Figure 1 is proposed for distributed autonomous multi-agent operations such as intelligent transport system. We assumed a fleet of transportation vehicles, known as agents, that operates in a workplace just as what the immune system does. The immune system is a collection of immune cells, tissues and molecules with different functionalities that mediate resistance to infections [6]. Our framework consists of agents with various capabilities in dealing with different problems in a dynamic environment. Comparing with the human immune system, the agents and their duties as referred in our control framework are equivalent to the immune cells and antigens respectively. An agent specification is shown in Figure 1 with an architecture, which was introduced in [8], detailing the autonomous operational mechanism based on the biological metaphor.

In the AIS-based control, agents are assumed to have no knowledge and experience in operating in the workplace in the beginning. In general, all agents under this AIS-based control framework have the ability to detect the surrounding environment within its sensory circle, and exchange information or communicate with other agents that are in close proximity defined by the communication circle. Each agent has different capability sets that determine the level of intelligence in tackling various tasks. Each task, on the other hand, has a complexity chain to signify what capabilities are essential to complete the task.

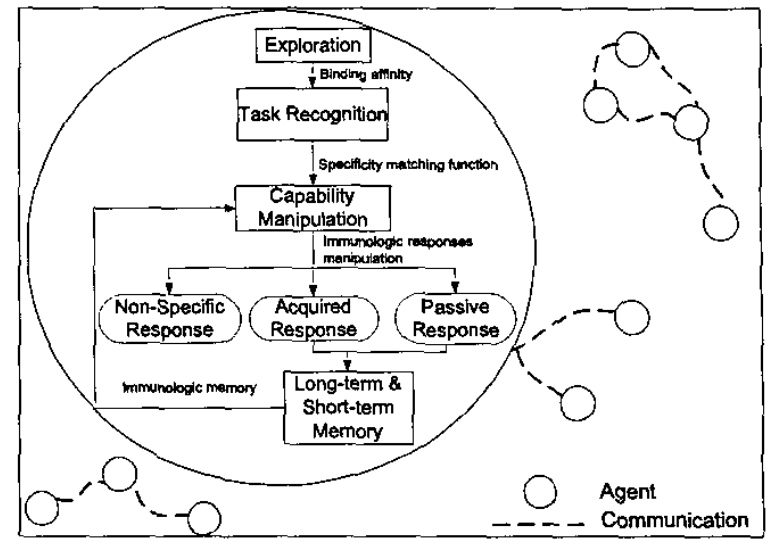

Figure 1. An AIS-based intelligent transport system

Upon deployment in a workplace, agents will explore the surrounding environment by undergoing pseudo-random motions. The agent experiences the workplace and decides its responses by perception of the environment within its sensory circle. The means of exploration allows agents to identify non-self and thus approach tasks. The AIS agents employ binding affinity [9] in task recognition to identify and approach targeted tasks. The binding affinity is enumerated by the distance between an agent and a particular task, frequency of task occurrence and agent familiarity with such a task. This mechanism for distinguishing between self and non-self is the prime function of the immune system. By discriminating the non-self, immune cells carry out pattern recognition by their receptors to identify a particular type of antigens [6], [7]. Specificity matching function [9] introduced in our AIS control framework is an analogue to the recognition of antigens structurally. This function aims to verify the feasibility of an agent to handle a particular task through string matching of its capability set with the task complexity chain.

A vehicle-based transport system performs a number of task components such as handling, distributing, loading and towing of material. The AIS control framework intends to classify each task components into different unique categories. In relation to the human immune system, three responses have been identified that allow AIS agents to manipulate different responses to cope with various problems. These responses include non-specific, acquired and passive responses [8]. Non-specific responses in general deal with simple and frequently encountered tasks. Acquired response is essential to specific and distinct tasks that need special talents and more advanced skills. Passive response is to deal with cooperative work through communication among the agents. The acquired skills and information obtained from capability manipulation or communication is stored in either short-term or long-term memory. 


\section{The immunity-based strategic behavioral control}

The aim to develop an intelligent transport system based on the AIS novel is its ability and efficiency in solving complicated problems. The immune sysiem composed of simple agents demonstrate complex collective behavior. These immune agents operate and cooperate in a fully non-deterministic and distributed manner. To develop such a decentralized system, it: is crucial to understand the mechanisms of the immune agents in fighting against antigens. We therefore develop an intelligent transport system, based on the AIS control framework, with agents having the behaviors inspired by the immune cells.

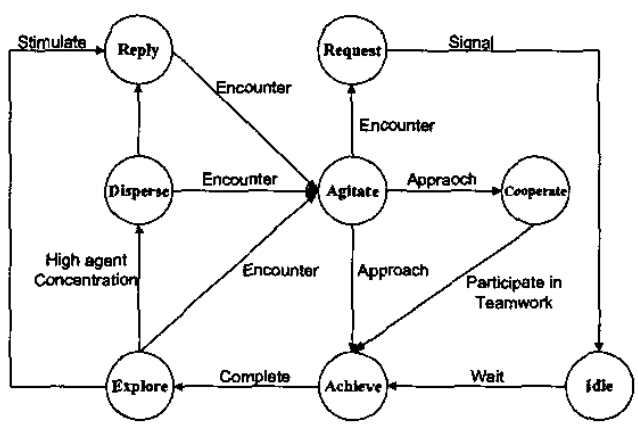

Figure 2. A state transition diagram of individual agent

The AIS agents are employed with full autonomy in decision-making and communication. They imitate the behaviors of immune cell in achieving goals whose individual elements are very simple. The internal behaviors of these AIS agents are determined by their perception of the environment, and such behaviors characterize their strategies in performing various operations. The state transition diagram as shown in Figure 2 defines the behaviors of an individual AIS agent for determining responses or movements strategically in various situations. Different behavioral states for the AIS agents which are derived based on the immune cells' behaviors are identified below:

- Explore state - Explore state describes an agent exploring the environment and searching for jobs in a random manner. Agent explores the surrounding environment within its sensory range by undergoirig pseudo-random motions. The new information, such as task location, that detected in this state will be store in short-term memory until they have been tackled. During this state, agent mainly searches for tasks around. It gairs knowledge of the environment through sensing and communicating with other agents. Besides discovering, an agent is in an active mode and is always ready to tackle tasks. If no immediate tasks to be tackled are detected, an agent in the explore state is ready to join cooperative work when a request signal is sent by other agents through communication.
- Disperse state - In biological immunity, an immune cell will stimulate other cells in response to a particular kind of antigen upon antigen recognition. When the concentration of the responded cells is too high, special signals will then be sent to suppress the activated cells in order to balance and regulate the antibody concentration [7]. According to this concept, a wandering zone is defined as a region where no tasks can be located or detected within that area with respect to the location of an agent. Concentration constraint is therefore applied in this zone to disperse the number of agents. A separationdistance is kept among agents to enhance the searching efficiency in the exploration stage. Figure 3 shows the wandering zone of Agent A. As an example, if the concentration constraint of the system is two agents, when the number of agents within Agent A's wandering zone is four as illustrated in Figure 3, Agent A will therefore try to keep away from the other agents to attain the concentration constraint.

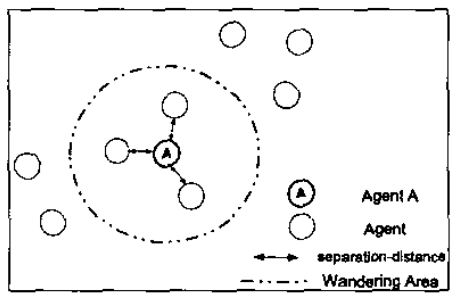

Figure 3. Wandering zone of agent $\mathrm{A}$

- Agitate state - Agitate state indicates an agent is stimulated by a job or other agents around its local area and hence the agent moves toward such stimulation signals. During exploration, agent will be stimulated by all the jobs within its sensory range. An agent determines which of those detected jobs should approach by comparing their binding affinity. The higher is the affinity, the greater the chance an agent would approach such a job. Hence, in the Agitate state, an agent is stimulated by and moves toward a goal either through detection or via communication. Once a situation has been ratified or a goal being achieved, an agent is in an aggressive mode and can no longer be activated by other stimulations.

- Achieve state - Achieve state indicates that an agent is handling and completing a job. After this state, the goal will be achieved and deleted from the agent's memory.

- Idle state - Some of the tasks cannot be solely handled by a single agent and they required cooperation among a number of agents. Since agents operate independently in an AIS-based system, there is no central controller that schedule and plan the agents' maneuver to perform a task. When an agent encounters a cooperative work, it will become idle and wait for other agents help.

- Request state - Request allows an agent who is in the Idle state to send signals asking for help. This request 
signal is a stimulation to activate other agents who are in the Explore and Disperse states. As mentioned above, an AIS-based system does not operate in a centralized manner, agents gain information mainly through sensing and communicating with each other. Hence, no central plans or strategies are defined to control the cooperative work in the AIS-based system. Agents cooperate by demonstrating complex collective behavior which is entirely emerged from communications.

- Reply state - Reply state describes an agent who receives a stimulation signal that is sent by others. This agent is then activated by the signal and reply to the sender by offering its help.

- Cooperate state - Cooperate state signifies an agent is participating in a cooperative work. Only successful cooperation can lead to the entering of the Cooperate state. If an agent has replied to a stimulation signal, this does not imply that a successful cooperation has been achieved until the agent has joined in the teamwork and started to work with others.

Through the above behavioral states derived from human immunity, agents are able to recognize the strategic plans of each other. Mutual understanding and agreement between agents can be achieved by exhibiting such strategic behavioral control based on their corresponding behavioral states. Hence, a control framework that achieves superior performance in terms of flexibility, scalability, robustness and self-organization of a multi-agent system implementing a truly automated transport system of the future can be developed.

\section{Simulations}

In addition to describing the theoretical development of the immune strategic behavioral control framework, a simulation study of an intelligent transport system operated by a fleet of AIS agents in a warehousing environment is undertaken. The simulation study examines the significance of each behavioral state and the impacts of these states on the overall performance of the transport system. These behaviors generate complex strategies to perform complicated operations in a nondeterministic manner. The strategic control is categorized into three main areas, which are exploration, achievement and cooperation, to testify how simple plan is evolved into a complicated operation by our strategic behavioral control framework.

The performance of the AIS-based multi-agent transport system is demonstrated in three different aspects. The AIS agents carry out material handling operations of searching and completing different tasks located all over the warehouse. The first simulation, Case 1 , includes simple tasks with the same complexity level that can be handled by non-specific response. Contrarily, the second simulation, Case 2 , tasks are more complicated and have different complexity levels. These tasks need to be completed by the acquired response where agents will gain new knowledge by capability manipulation upon task completion. The third simulation, Case 3 , highlights the cooperation ability of the immune strategic behavioral control. In this case, all tasks involve teamwork. A group of agents is therefore required to achieve a goal by cooperation.

\subsection{Overall efficiency}

The significance of the different behavioral states is illustrated in Figure 4. These behavioral states determine the primitive behaviors of the AIS agents. Agents perform these basic strategies to achieve goals in all situations. The results of Figure 4 are measured in number of state occurrence per agent. These results indicate the Explore state is the most significant state among the others. Since agents that are deployed to the warehouse with no prior information regarding the environment and tasks, exploration is the fundamental state that enables the agents to learn about and search tasks within the environment. In addition, through exploring the environment, an agent gains extra information through communicating with other agents. Communication is therefore the second important characteristic in the AISbased system.

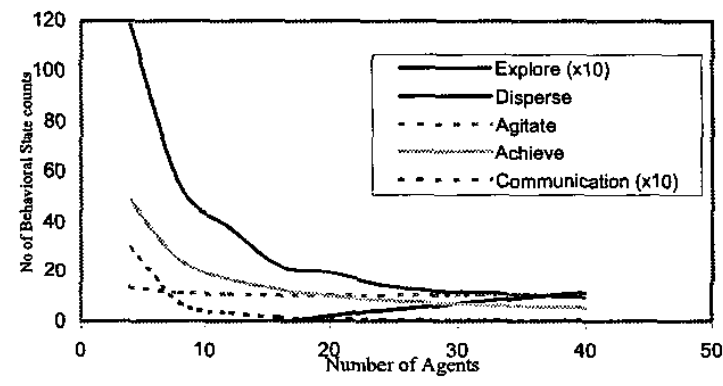

Figure 4. Significances of each behavioral state

The importance of exploration and communication are far more than the other states. This indicates that AISbased agents spend most of their time in searching for tasks and learning their workspace. That is an ordinary phenomenon for a non-deterministic distributed multiagent system. While the number of exploration and communication are greater than the other states, existence of a steady state situation of the AIS-based transport system can be found. As shown in Figure 4, all the primitive behaviors become steady when the number of agents employed in the system is more than twenty. The degree of exploration and communication has also significantly decreased when the steady condition is approached. A saturation point where an optimal number of agents required for effective operation using the AISbased control framework can be derived.

Figure 5 further illustrates the number of steps taken by each AIS agent through responses manipulation for the three cases described above. Similar to Figure 4, steady 
states are obtained in all cases as shown in Figure si. A noticeable decrease in the number of steps taken by the agents is observed. The first two cases have a better performance than the last case. This is because in Case 3, agents need to participate in teamwork which requires more effort to complete all the tasks. On the other hand, the performances of the first two cases show similar results. Case 1 consists only simple tasks that can be handled directly by non-specific response whereas Case 2 consists complex and difficult tasks. They require agents to gain new capability in order to be tackled. This justifies the efficiency of the responses manipulation in acquiring new knowledge to handle unfamiliar task by our AISbased control is attained. Hence, the proposed transport system based on an AIS approach has the flexibility and efficiency of a multi-agent system with simple behaviors that exhibits complex strategies.

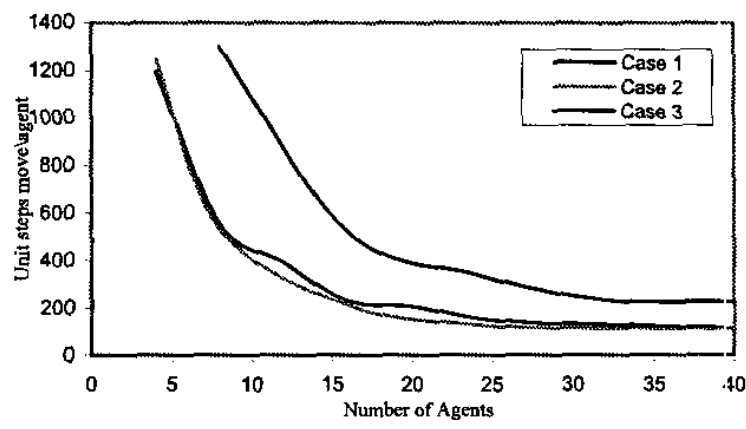

Figure 5. Performances of the AIS-based transport system

\subsection{Exploration}

Explore and Disperse states are the two main behaviors that govern exploration. Figure 6 shows the interrelationship of these two states for the three cases studied. The average number of state occurrence per agent is taken to testify how exploration is evolved from the Explore and Disperse states when agents deal with different types of problems presented in a workplace. A direct proportional trend is resulted in Case 1 and 2 between the Explore with Disperse states and Disperse states with the number of agents deployed when all jobs are done. This is a phenomenon to which no cooperative task is existed. As the number of agents increased, dispersion would take place more frequently. Hence, the number of occurrence of the Explore state is decreased when the number of agents and Disperse state increased.

As cooperation is required in Case 3, the concentration of agents within the same proximity is much higher. In this case, dispersion is more essential to scatter the agents once they have completed a cooperative task in order to maintain the exploration efficiency. The amount of the Disperse state in this case is therefore much higher than the Explore state even with small number of agents deployed. Hence, the Disperse state is an essential behavior to avoid overcrowding and allow efficient exploration activity.

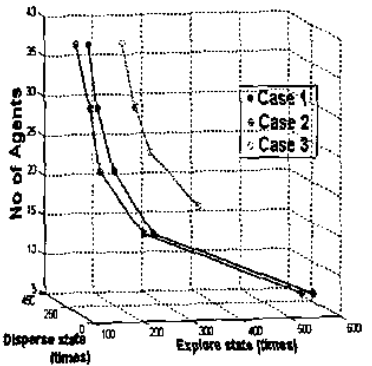

Figure 6. Behavioral states for exploration

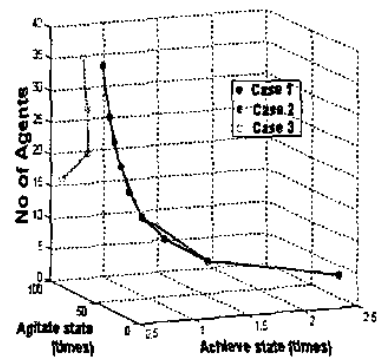

Figure 7. Behavioral states for achievement

\subsection{Achievement}

In achieving goals, AIS agents act aggressively and adopt the Agitate state once they have detected a task. An agent will then complete the task in the Achieve state. The average number of state occurrence per agent is taken in Figure 7 to indicate how agents behave in achieving goals and how often an agitated agent can successfully tackle a targeted task in the three different situations.

In the first two cases, agents achieve goals only through responses manipulation. A straightforward sequence of agitate when a task is found, achieve the targeted task and complete the task is resulted. Hence, the results for Case 1 and 2 have a similar trend where some of the points are almost overlapped. With an increasing number of agents deployed, the number of Achieve and Agitate states that each agent comes across is therefore decreased. This is mainly due to the increase in workforce where the number of tasks completed per agent is reduced. Moreover, the amounts of the Agitate and Achieve states are proportional to each other. This implies that the achievement rate of non-cooperative task depends on the agent searching ability to look for a goal as the agent will become agitated once a task is found.

Case 3 on the other hand requires cooperation between agents; therefore the number of Agitate state that an agent comes across is much greater than the number of Achieve state. As a group of agents is essential to handle one task, the number of Agitate state is therefore increased significantly. This also leads to a drop in the achievement rate per agent and hence the number of occurrence of the Achieve state in Case 3 is much lower when compared to Case 1 and 2.

\subsection{Cooperation}

Reply, Request, Idle and Cooperate states are the main behaviors evolved into a cooperative work. Figure 8 illustrates the results of the interrelationships of Request with Reply states and Idle with Cooperate states. The two curves show a very similar result. An agent who is handling a task that required joint effort will become idle if the number of workers is not enough. In the meantime, the agent will send signals to request for assistance. The numbers of Idle and Request states are therefore almost the same. On the other hand, the number of Reply state is 
greater than the number of Cooperate state. This is because agents who received signal requesting for help during exploration will reply to the signal, move forward to the task and cooperate with other agents to complete the task. As a cooperative task needs to be completed by at least two agents, the number of Reply state is therefore more than the number of Cooperate state. Also, a reply to a signal does not imply a successful cooperation.

A reduction of agents being idle and request for help is resulted when the number of agents is increased (Figure 8). There is a noticeable difference in the rate of decline of the number of agents when twenty-four agents are deployed. This reveals the optimal number of agents necessary for effective cooperation in our simulated AISbased system. According to the result, a significant improvement is observed when the number of agents employed is greater than twenty-four. A steady improvement is also obtained beyond the optimal point which is mainly due to the constant increase of workforce. We can conclude that the AIS-based behavioral control is able to obtain effective cooperation with a certain number of agents deployed. An optimal solution, which may differ for various systems, indicates the most favorable number of agents required is therefore predictable. This in turn can prevent any shortage or wastage of input which hinder the system overall performance and efficiency.

Figure 9 illustrates the peaks measured in the number of occurrence of the Cooperate state in relation to the Request and Reply states. Request and Reply states are treated as input parameters. The results are obtained by translating and scaling Gaussian distributions with the two variables which demonstrates the proportion of time that an agent should participate in performing cooperative task. The maximum number of Cooperate state occurs when the probability of request is almost zero and reply is approximately 0.3 . This implies that for effective cooperation, AIS agents only spend about one third of their time joined in teamwork. They have high adaptability in handling different kinds of job and accommodating with other collaborators. Under the AISbased control, agents are functioning with great flexibility. Hence, coalition can be resulted with no requirement of centralized coordination.

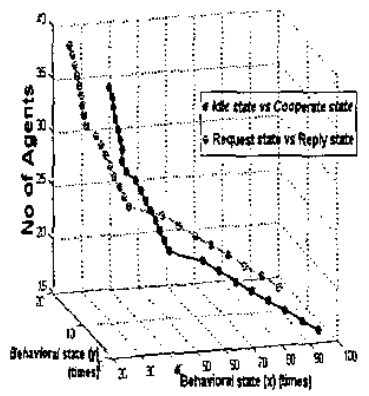

Figure 8. Behavioral states for cooperation state in performing

\section{Conclusion}

This paper presents a distributed control framework based on the biological theory of immunology to manage, coordinate and schedule a fleet of agents employed in an automated transport system. Different behavioral states for the AIS agents that are derived based on the behavior of human immune cells have been identified to describe the strategies of the agents. The internal behaviors of the AIS agents, which are decided by their perception of the environment, are studied in detail to describe their strategies in performing various operations. These operations include cooperation among agents that are driven by the communication and self-regulation concepts of the human immune mechanisms.

In addition to describing the theoretical development of the immune strategic behavioral control framework, the paper presents a simulation study of an intelligent transport system operated by a fleet of AIS agents in a warehousing environment. The simulation study aimed to examine the significance of each behavioral state in every necessary action or step and the impacts of these states to the overall performance of the transport system. The results of the simulation illustrated how the immune strategic behavioral-based control enhances the system overall efficiency.

\section{References}

[1] P. M. Lydyard, A. Whelan and M.W. Fanger, Instant notes in immunology, Oxford UK, Bios, Springer 2000.

[2] J. Kim and P. Bentley, The Human Immune System and Network Intrusion Detection, 7th European Conference on Intelligent Techniques and Soft Computing, Aachen, 1999.

[3] J.H. Jun, D.W. Lee and K.B. Sim, Realization of cooperative strategies and swarm beharior in distributed autonom ous robotic systems using artificial immune system, IEEE SMC, Vol. 6, pp. 614-619, Oct 1999.

[4] S. Sathyanath and F. Sahin, AISIMAM - An Artificial Immune System Based Intelligent Multi Agent Model and its Application to a Mine Detection Problem, ICARIS 2002, Session I, pp. 22-31.

[5] R.O. Canbam and A.M. Tyrrell, A Multilayered Immune System for Hardware Fault Tolerance within an Embryonic Array, ICARIS 2002, Session I, pp. 3-11, 2002.

[6] K. D. Elgert, Immunology: understanding the immune system, New York: Wiley-Liss, 1996.

[7] L.J. Eales, Immunology for life scientists: $A$ basic introduction: A student learning approach, Chichester, John Wiley \& Sons, 1997.

[8] H.Y.K. Lau and W.K.V. Wong, Immunologic Control Framework for Automated Material Handling, ICRAIS 2003, pp. 57-68, 2003.

Figure 9. The probability of the occurrence of an agent's cooperative tasks
[9] H.Y.K. Lau and W.K.V. Wong, Immunologic Response Manipulations for AIS Agent, ICRAIS 2004 (in printed). 